\title{
The Application of Resonance-Enhanced Multiphoton Ionization Technique in Gas Chromatography Mass Spectrometry
}

\author{
Adan Li, Jianzheng Song, Yang Sun, and Tifeng Jiao \\ Hebei Key Laboratory of Applied Chemistry, School of Environmental and Chemical Engineering, Yanshan University, \\ Qinhuangdao 066004, China
}

Correspondence should be addressed to Adan Li; adanli@ysu.edu.cn

Received 23 March 2014; Accepted 27 April 2014; Published 12 May 2014

Academic Editor: Xinqing Chen

Copyright (C) 2014 Adan Li et al. This is an open access article distributed under the Creative Commons Attribution License, which permits unrestricted use, distribution, and reproduction in any medium, provided the original work is properly cited.

Gas chromatography resonance-enhanced multiphoton ionization time-of-flight mass spectrometry (GC/REMPI-TOFMS) using a nanosecond laser has been applied to analyze the 16 polycyclic aromatic hydrocarbons (PAHs). The excited-state lifetime, absorption characters, and energy of electronic states of the 16 PAHs were investigated to optimize the ionization yield. A river water sample pretreated by means of solid phase extraction was analyzed to evaluate the performance of the analytical instrument. The results suggested that REMPI is superior to electron impact ionization method for soft ionization and suppresses the background signal due to aliphatic hydrocarbons. Thus, GC/REMPI-TOFMS is a more reliable method for the determination of PAHs present in the environment.

\section{Introduction}

The ionization technique for the analyte molecules is of particular importance to mass spectrometry (MS); the application of varied ion sources of the MS introduces selectivity features of the analysis result, that is, species, isomers, or state-selective ionization and control of fragment intense [1-3]. Electron impact (EI) represents one of the most useful ionization methods used in the current studies of gas chromatography/mass spectrometry (GC/MS) analysis. The method, however, suffers from the mass information caused by the hard ionization, which results in the difficulty of identifying the complicated matrix of samples. The typical soft ionization including chemical ionization (CI), field ionization (FI), and photoionization (PI) has been developed to overcome the weakness of the hard ionization method. PI is superior to CI and FI on the mass spectrometric analysis because there is no limitation caused by the chemical reaction in CI and oxidation of the emitter in FI. Laser based PI methods, such as resonance-enhanced multiphoton ionization (REMPI) and single-photon ionization (SPI), have been very successfully applied to research and practical applications [4-7]. REMPI is more elective and sensitive than
SPI for the ionization of aromatic hydrocarbons using UV wavelengths that are readily accessible with standard pulsed lasers. Most applications of REMPI-MS involve direct-inlet MS, but successful couplings of REMPI-MS to GC and LC as well as laser desorption have also been reported [5, 7]. The coupling of such techniques with mass-spectrometry has expanded considerably the realm of analytical capabilities of MS. A laser with different pulse durations has been employed for ionization [8-10]. For example, a femtosecond laser has been successfully used for efficient ionization before relaxation process of internal conversion and intersystem crossing of some chlorinated, brominated compounds [11,12]. Unfortunately, a high cost, a large dimension, and difficulties in the maintenance prevent practical use of this method in environmental analysis. In addition, if the laser pulse width is much shorter than the time scale of relaxation, the use of such high intensive laser leads to unfavorable dissociation of both neutrals and ions, thus making sensitive and reliable analysis more difficult [13]. To date, a number of studies have been reported to investigate the importance of laser parameters on the ionization yield for various chemical species $[9,10,14-16]$.

In this study, a combination of gas chromatography (GC) and REMPI/MS (GC-REMPI/MS) using a nanosecond laser 
was utilized for trace analysis of 16 polycyclic aromatic hydrocarbons (PAHs) in the priority list of US Environmental Protection Agency (EPA) to investigate the best performance of this technique. With REMPI, the molecule absorbs the first photon for excitation and the second photon for subsequent ionization. Since only a molecule absorbing the first photon can be ionized, interference arising from aliphatic hydrocarbons can be reduced. Thus, this technique provides superior selectivity since each congener can be selectively ionized via resonance excitation. A river water sample was also analyzed after pretreatment by solid phase extraction (SPE) method using this system to demonstrate the advantage especially in selectivity in environmental analysis.

\section{Materials and Methods}

2.1. Apparatus. Figure 1 shows the experimental setup in this study. The fourth harmonic emission of a Nd:YAG laser (Crylas, $266 \mathrm{~nm}, 1 \mathrm{~ns}, 1 \mathrm{kHz}$ ) was employed as an ionization source. One $\mu \mathrm{L}$ of the analyte was injected into a GC system (Agilent Technologies, 6890N) using an autosampler (Agilent Technologies, 7683B) followed by a DB-5 $(30 \mathrm{~m} \times 0.32 \mathrm{~mm}$ I.D.) capillary column. Helium was used as a carrier gas at a constant flow rate of $1 \mathrm{~mL} / \mathrm{min}$. The temperature program for analysis of PAHs was set at a rate of $20^{\circ} \mathrm{C} / \mathrm{min}$ from 40 to $120^{\circ} \mathrm{C}$. It was further increased at a rate of $5^{\circ} \mathrm{C} / \mathrm{min}$ from $120^{\circ} \mathrm{C}$ to $250^{\circ} \mathrm{C}$ and held for $3 \mathrm{~min}$ and was then increased at a rate of $5^{\circ} \mathrm{C} / \mathrm{min}$ to $280^{\circ} \mathrm{C}$ and held for $10 \mathrm{~min}$. The temperatures of the injection port and the transfer line were maintained at $300^{\circ} \mathrm{C}$. The sample eluting from GC was introduced into a linear-type TOF-MS as an effusive molecular beam. A microchannel plate (Hamamatsu, F4655-11) was utilized for the detection of the ions induced by multiphoton ionization. The signal of the mass spectrum was optimized using a digital oscilloscope (Tektronix DPO7104, $1 \mathrm{GHz}, 20 \mathrm{GS} / \mathrm{s}$ ). The twodimensioned data of GC/REMPI-TOFMS was recorded by a digitizer (Agilent Technologies, 8 bit PCI High-speed Signal Analyzers, Acqiris AP240, $1 \mathrm{GHz}, 1-2 \mathrm{GS} / \mathrm{s})$. The final results of the data were analyzed and displayed using Labview software.

2.2. Reagents. A standard mixture of PAHs in the priority list of US EPA (acenaphthene (ACE), acenaphthylene (ACY), anthracene (ANT), benzo(a)anthracene (BaA), benzo(b)fluoranthene $(\mathrm{BbF})$, benzo(k)fluoranthene $(\mathrm{BkF})$, benzo(ghi)perylene (BPY), benzo(a)pyrene (BaP), chrysene (CHR), dibenzo(a,h)anthracene (DBA), fluoranthene (FLT), fluorene (FLU), indeno(1,2,3-cd)pyrene (IND), naphthalene (NAP), phenanthrene (PHE), and pyrene (PYR)) prepared at a concentration of $2000 \mu \mathrm{g} / \mathrm{mL}$ in methylene chloridebenzene $(1: 1 \mathrm{v} / \mathrm{v})$ and a mixture of deuterated internal standards (I.S.) (acenaphthene- $\mathrm{d}_{10}$, phenanthrene- $\mathrm{d}_{10}$, and chrysene- $d_{12}$ ) prepared at a concentration of $500 \mu \mathrm{g} / \mathrm{mL}$ in acetone were purchased from Supelco (Bellefonte, PA, USA). All the solutions were stored in containers made of amber glass at $4^{\circ} \mathrm{C}$. Analytical-reagent grade of acetonitrile, acetone, methanol, and dichloromethane was purchased from Kanto

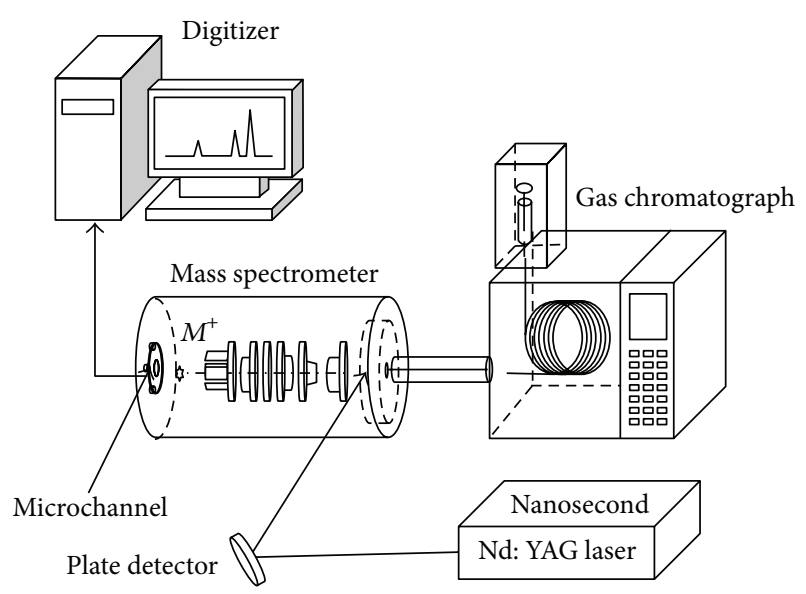

FIGURE 1: Experimental setup of the GC/REMPI-TOFMS based on the forth harmonic emission of a nanosecond Nd:YAG laser.

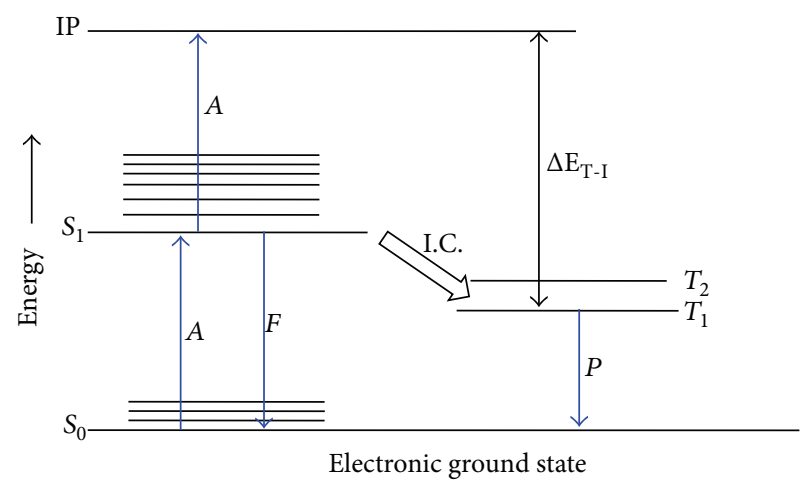

FIGURE 2: Schematic diagram of one-color two-photon ionization process and the relaxation process. A: photon absorption; F: fluorescence; S: singlet state; P: phosphorescence; I.C.: intersystem crossing.

Kagaku (Tokyo). Deionized water was obtained from a Milli$\mathrm{Q}$ water purification system (Millipore, Molsheim, France).

2.3. SPE. A surface water sample was collected from a river located in the northern part of Kyushu area in Japan. A river water sample was spiked with $0.5 \mathrm{~mL}(200 \mathrm{pg} / \mu \mathrm{L})$ of I.S. to investigate the recovery of PAHs in the SPE process. The $500 \mathrm{~mL}$ of water sample was passed through an SPE cartridge (Sep-Pak Plus C18 with $10 \mathrm{~mL}$ of dichloromethane, $10 \mathrm{~mL}$ of methanol, and $10 \mathrm{~mL}$ of Milli-Q water) at a flow rate of $10 \mathrm{~mL} / \mathrm{min}$. The PAHs and dioxin were eluted with $5 \mathrm{~mL}$ of dichloromethane from the Sep-Pak Plus C18 cartridge connected with the Sep-Pak Dry cartridge. The solvent of the extract was evaporated under a nitrogen flow and filled with $0.5 \mathrm{~mL}$ of acetonitrile for analysis by GC/REMPI-TOFMS.

\section{Results and Discussion}

3.1. Excited-State Lifetime. Figure 2 shows a total ion chromatogram (TIC) of the standard mixture sample that contained 16 PAHs $(200 \mathrm{pg} / \mu \mathrm{L})$. Generally, the excited-state lifetimes $\left(\tau_{s}\right)$ have an important influence on the ionization 


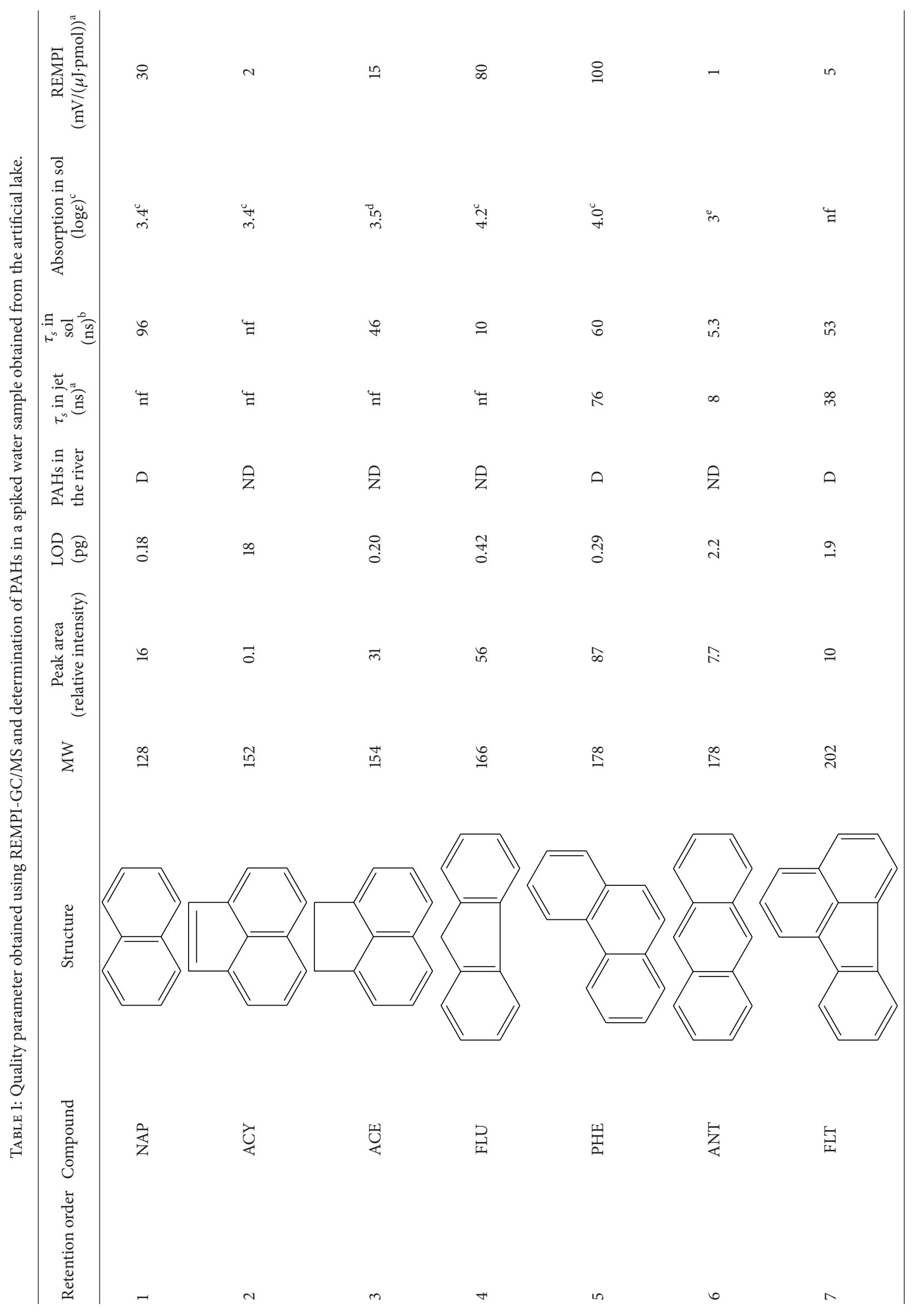




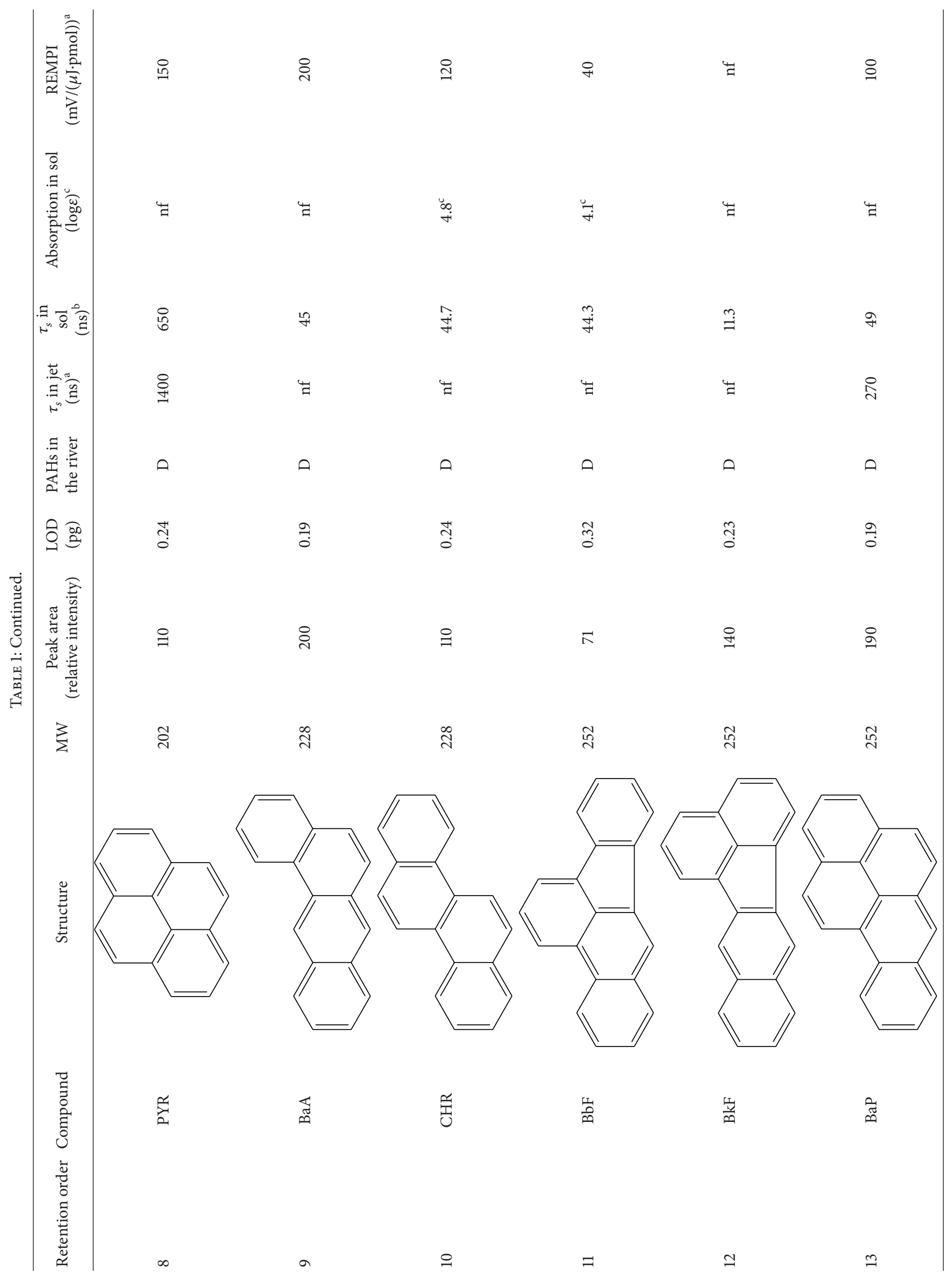




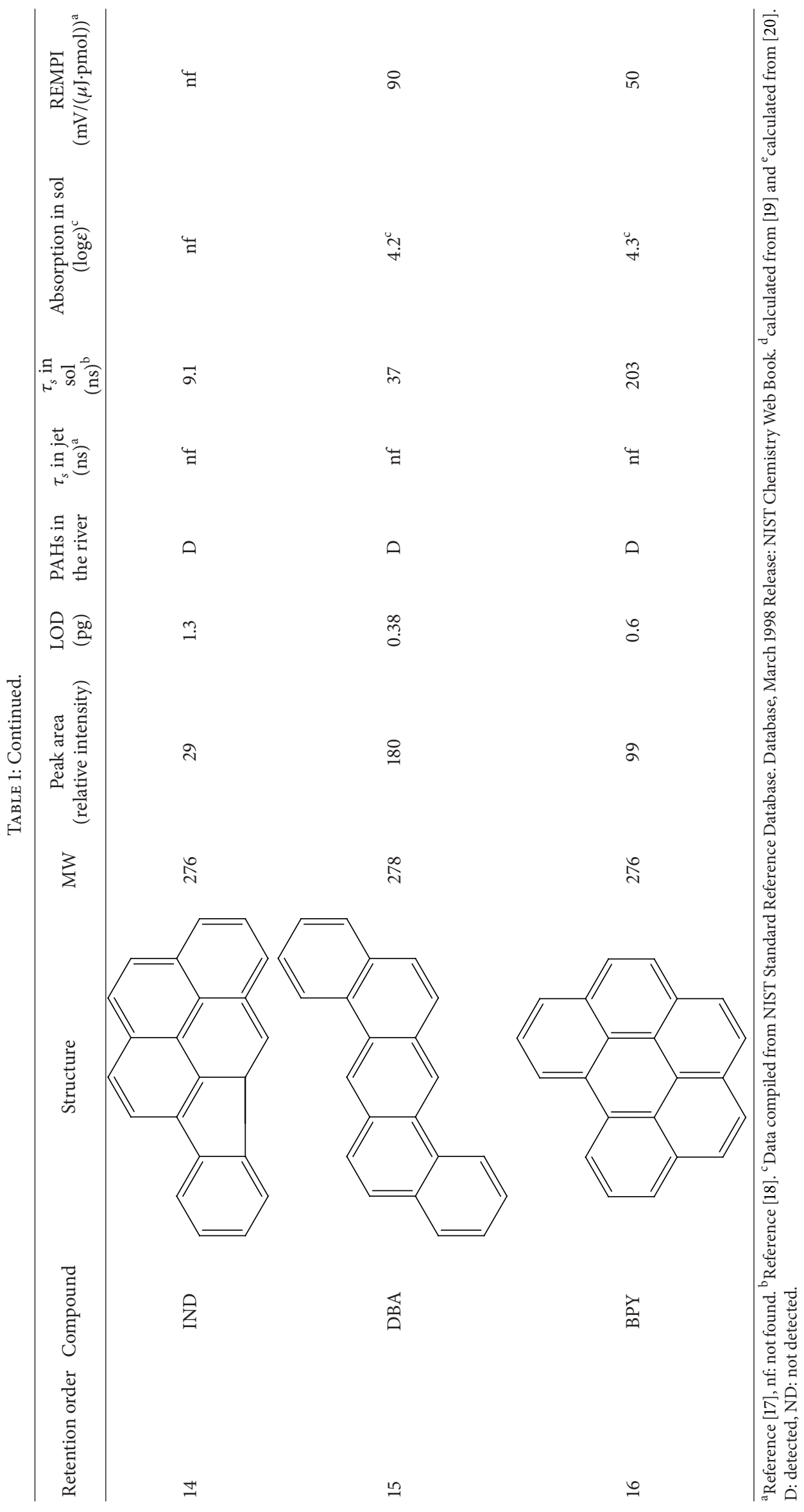


TABLE 2: Electronic states energy of the 16 PAHs in U.S. EPA list.

\begin{tabular}{|c|c|c|c|c|c|c|c|c|c|c|c|c|c|c|c|c|}
\hline Compound & NAP & $\mathrm{ACY}$ & ACE & FLU & PHE & ANT & FLT & PYR & $\mathrm{BaA}$ & CHR & $\mathrm{BbF}$ & $\mathrm{BkF}$ & $\mathrm{BaP}$ & IND & DBA & BPY \\
\hline $\mathrm{IP}(\mathrm{eV})$ & $8.12^{\mathrm{a}}$ & $8.22^{\mathrm{a}}$ & $7.68^{\mathrm{a}}$ & $7.88^{\mathrm{a}}$ & $7.90^{\mathrm{a}}$ & $7.44^{\mathrm{a}}$ & $7.9^{\mathrm{a}}$ & $7.43^{\mathrm{a}}$ & $7.53^{\mathrm{a}}$ & $7.6^{\mathrm{a}}$ & $7.70^{\mathrm{b}}$ & $7.48^{\mathrm{b}}$ & $7.10^{\mathrm{a}}$ & $\mathrm{nf}$ & $7.38^{\mathrm{a}}$ & $7.16^{\mathrm{a}}$ \\
\hline$S_{1}(\mathrm{eV})^{\mathrm{c}}$ & $4.03^{\mathrm{d}}$ & $\mathrm{nf}$ & $\mathrm{nf}$ & $4.12^{\mathrm{c}}$ & $3.61^{\mathrm{d}}$ & $3.31^{\mathrm{c}}$ & $3.06^{\mathrm{c}}$ & $3.34^{\mathrm{c}}$ & $3.22^{c}$ & $3.44^{\mathrm{c}}$ & $3.38^{\mathrm{c}}$ & $3.10^{\mathrm{c}}$ & $3.08^{\mathrm{c}}$ & $\mathrm{nf}$ & $3.14^{\mathrm{c}}$ & 3.05 \\
\hline$T_{1}(\mathrm{eV})^{\mathrm{c}}$ & $2.88^{\mathrm{d}}$ & $\mathrm{nf}$ & $\mathrm{nf}$ & $2.95^{\mathrm{c}}$ & $2.7^{\mathrm{d}}$ & $1.85^{\mathrm{c}}$ & $2.92^{\mathrm{c}}$ & $2.11^{\mathrm{c}}$ & $2.07^{\mathrm{c}}$ & $2.48^{\mathrm{c}}$ & $2.49^{c}$ & $2.19^{c}$ & $1.84^{\mathrm{c}}$ & $\mathrm{nf}$ & $2.26^{\mathrm{c}}$ & 2.00 \\
\hline$E_{T-I}(\mathrm{eV})$ & 5.24 & $\mathrm{nf}$ & $\mathrm{nf}$ & 4.93 & 5.2 & 5.59 & 4.98 & 5.32 & 5.46 & 5.12 & 5.21 & 5.29 & 5.26 & $\mathrm{nf}$ & 5.12 & 5.16 \\
\hline
\end{tabular}

${ }^{a}$ Data compiled from NIST Standard Reference Database. Database, March 1998 Release: NIST Chemistry Web Book; nf: not found. ${ }^{b}$ Reference [21]. ${ }^{c}$ calculated from [22] and ${ }^{\mathrm{d}}$ calculated from [23].

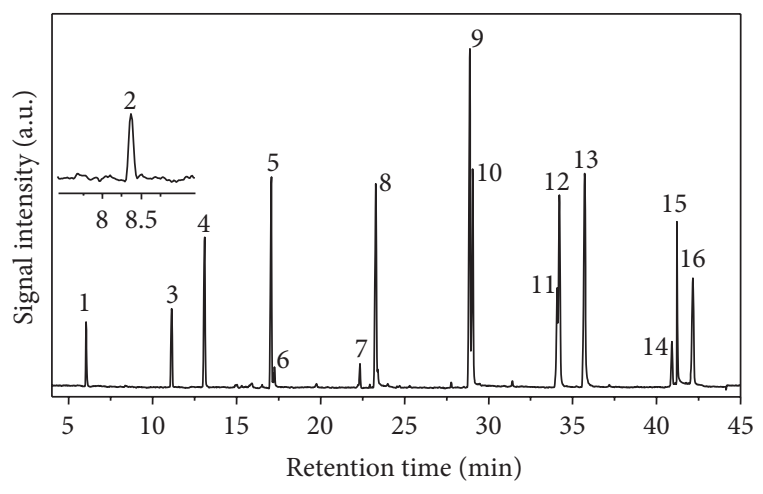

FIGURE 3: The total ion chromatogram (TIC) of GC/REMPI-TOFMS obtained for a standard solution of PAHs at $200 \mathrm{pg} / \mu \mathrm{L}$. Peak numbers are 1, NAP; 2, ACY; 3, ACE; 4, FLU; 5, PHE; 6, ANT; 7, FLT; 8, PYR; 9, BaA; 10, CHR; 11, BbF; 12, BkF; 13, BaP; 14, IND; 15, DBA; and 16, BPY.

efficiency of PAHs, because an important feature of the PAHs molecules considered is the close-to-unity sum of the quantum yields of fluorescence $\Phi_{F}$ and of triplet formation $\Phi_{T}$ due to the intersystem crossing transition $S_{1} \rightarrow T_{1}$. The photophysical properties of the PAHs and the experimental results are shown in Table 1 . There are only a few reports on the lifetime of isolated PAHs in the gas phase, although the lifetimes of PAHs in the condensed phase are well known [17]. The lifetimes of PAHs in solution are similar to, or slightly shorter than, those in the gas phase due to an additional relaxation pathway from collisions between the analyte and solvent molecules [18]. Therefore, the lifetimes of the 16 PAHs (8-1400 ns in the gas phase and 6-650 ns in the condensed phase, except for ACY and IND) were much longer than the pulse width of the laser used in the present study ( $1 \mathrm{~ns})$. Thus, no obvious loss of ionization efficiency could be observed for most of the 16 PAHs except of ACY. In ACY molecule, the major deactivation pathway from the $S_{1}$ state was an efficient $S_{1} \rightarrow S_{0}$ internal conversion. The lifetime of the $S_{1}$ state of ACY had been determined to be 345 ps and 0.2 ns, which were shorter than the pulse width of the laser in the present study $[24,25]$. In the other cases, the $\tau_{s}$ was not the determination factor to the ionization efficiency. For example, the $\tau_{s}$ of PYR is the longest in all the analytes in this research, but the ionization efficiency of it is even lower than that of BaP. Thus, only when the $\tau_{s}$ of the PAHs is shorter than the pulse width of the laser, the ionization efficiency can be increased using a more intensive laser.
3.2. Energy of Electronic States. The schematic diagram of the photochemical conversion in the one-color two-photon ionization process is shown in Figure 3. When the gas molecules absorb photons, the electrons can be elevated to higher energy state to form the excited state. The PAHs molecules having absorbed the first photon in the excited state could absorb another photon to the ionization potential (IP) to accomplish the $1+1$ REMPI process. Simultaneously, it could lose energy through the intersystem crossing or the internal conversation to the triplet state or the ground state because the crossing and conversation from the upper to the lower excited state are normally fast and very efficient; the states mentioned here are the lowest singlet-state $S_{1}$ and lowest triplet-state $T_{1}$. The photochemistry properties of the 16 PAHs are shown in Table 2. The energy from triplet state to IP was shown as $E_{T-I}$ in Table 2. It should be noticed that, in the case of ANT, $S_{1}$ may cross to $T_{2}$, which is nearly isoenergetic with $S_{1}$ [26]. Thus, in ANT, a small energy gap and consequently a favorable Frank-Condon factor exist for intersystem crossing, thus leading to a higher limit of detection (LOD) $(S / N=3)$ of it. From Table 2, it was obvious that the two-photon energy applied in this study $(266 \mathrm{~nm}+$ $266 \mathrm{~nm}=9.32 \mathrm{eV}$ ) was qualified for a $1+1$ REMPI for all the 16 PAHs in this research, but one-photon energy could not provide the subsequent exciting from the triplet-state.

3.3. Absorption Character. During $1+1$ REMPI absorption process, the ionization efficiency of a molecule depended primarily on two factors: $\sigma_{1}$, the cross section for absorption of the first photon which excites the molecule from the ground electronic state into an electronically excited intermediate state; $\sigma_{2}$, the cross section for absorption of the second photon which pumps the excited molecule into the ionization continuum. In the theory when the first photon absorption event is the rate-limiting step of the ionization process (i.e., $\sigma_{1} \ll \sigma_{2}$ ), then similarities are expected between the absorption or fluorescence excitation spectra and the $1+1$ REMPI spectra. If, on the other hand, the second photon absorption event is the rate-determining step (i.e., $\sigma_{1} \gg \sigma_{2}$ ), then the spectroscopy of the molecules in the excited state is expected to dominate the spectrum. As to $\sigma_{1}$, it can be derived from the distinction coefficiency $(\varepsilon)$ as follows:

$$
\begin{gathered}
\sigma_{1}=2.303 \frac{\varepsilon}{N_{A}}=3.82 \times 10^{-21} \mathcal{E} \\
\left(N_{A}: \text { Avogadro constant }\right) .
\end{gathered}
$$




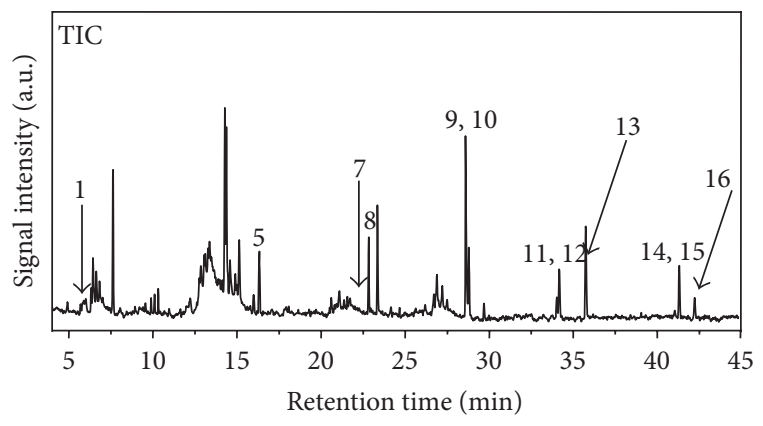

Figure 4: The TIC obtained by GC/REMPI-TOFMS from a river sample spiked with an I.S. standard mixture of PAHs. Peak numbers are 1, NAP; 5, PHE; 7, FLT; 8, PYR; 9, BaA; 10, CHR; 11, BbF; 12, BkF; 13, BaP; 14, IND; 15, DBA; and 16, BPY.

There were few reports about the gas phase UV absorption spectra of PAHs because in most cases the UV absorption spectra were given in solution phase. But the shape and width of these two phases were the same and they shift generally between 6 and $18 \mathrm{~nm}$ [27]. Thus the extinction coefficients absorption discussed here was the data obtained from the solution phase.

From the peak area of PHE and ANT, shown in Table 1, it was clear that the ionization efficiency of PHE was about 11 times that of ANT. The dominant ionization efficiency at $266 \mathrm{~nm}$ of PHE would change if a wavelength was more selective to ANT. For example, at $310 \mathrm{~nm}$, ANT was preferentially ionized in the presence of PHE due to the different $\sigma_{1}$ absorption character [28]. On the other hand, the $\sigma_{2}$ could determine the intensity of the REMPI spectra as well. As shown in Table 1, the $\varepsilon$ of NAP and ACY was the same, but the REMPI intensity of NAP was 15 times that of ACY, which resulted in the lower ionization efficiency of ACY compared to NAP.

3.4. Measurement of a Water Sample. A sample derived from surface water was spiked with three deuterated compounds, which served as internal standards, and was analyzed by GC/REMPI-TOFMS.The TIC was displayed by extracting the data between $m / z=100$ and 400 in Figure 4, in which 12 PAHs on the U.S. EPA list and the internal standards were evident. We noticed that several of the chromatograph peaks had $m / z$ values that were identical to those of the PAHs on the U.S. EPA list. These peaks might have been due to isomers of the PAHs. Therefore, the signal peaks should be carefully identified, even though the REMPI technique had superior selectivity. For example, several NAP isomers with the chemical formula $\mathrm{C}_{10} \mathrm{H}_{8}$ were detected, which might be either $1 \mathrm{H}-$ indene-1-methylene, azulene, or 2-methylene-2H-indene. A more serious case was observed for FLT; there were several peaks with larger signal intensities than that of FLT. These isomers could be either acephenanthrylene, aceanthrylene, or their analogues. However, for all the compounds detected in this research, the mass chromatogram was much simplified and generally no fragments except the parent ion could be detected. Compared to the conventional ionization technique of GC/EI-MS, the background of the mass spectra was much reduced and the selectivity was improved greatly.

\section{Conclusions}

The forth harmonic emission of a nanosecond Nd:YAG laser emitting at $266 \mathrm{~nm}$ was employed to detect 16 PAHs of US EPA both in standard samples and in a water sample. The detection limits of the $16 \mathrm{PAHs}$ were determined to be $0.18 \sim$ $18 \mathrm{pg}$. The absorption characters at $266 \mathrm{~nm}$ can influence the ionization efficiency obviously, owing to the longer lifetime of the 16 PAHs compared to the pulse width of the laser. As to ionization potential, the photon energy of the laser applied in this study is enough to induce the REMPI process but not an ionization process from triplet state. In the analysis of the river sample, few compounds except PAHs can be detected in the mass chromatogram due to selective ionization of REMPI. The GC/REMPI-TOFMS based on nanosecond laser ionization is suitable for the trace detection of PAHs in environmental sample due to the high selectivity and sensitivity.

\section{Conflict of Interests}

The authors declare that there is no conflict of interests regarding the publication of this paper.

\section{Acknowledgments}

This research was supported by the Science and Technology Research of Higher Education Institute of Hebei province of China (QN20131038) and the Independent Research Project for Yong Teachers of Yanshan University (13LGA014).

\section{References}

[1] T. Uchimura, Y. Sakoda, and T. Imasaka, "On-line concentration by analyte adsorption and subsequent laser desorption in supersonic jet spectrometry," Analytical Chemistry, vol. 80, no. 10, pp. 3798-3802, 2008.

[2] D. M. Lubman, "Optically selective molecular mass spectrometry," Analytical Chemistry, vol. 59, no. 1, pp. 31A-40A, 1987.

[3] F. Mühlberger, J. Wieser, A. Ulrich, and R. Zimmermann, "Single photon ionization (SPI) via incoherent VUV-excimer light: robust and compact time-of-flight mass spectrometer for on-line, real-time process gas analysis," Analytical Chemistry, vol. 74, no. 15, pp. 3790-3801, 2002.

[4] U. Boesl, H. J. Neusser, and E. W. Schlag, "Multi-photon ionization in the mass spectrometry of polyatomic molecules: cross sections," Chemical Physics, vol. 55, no. 2, pp. 193-204, 1981.

[5] A. Li, T. Uchimura, Y. Watanabe-Ezoe, and T. Imasaka, "Analysis of dioxins by gas chromatography/resonance-enhanced multiphoton ionization/mass spectrometry using nanosecond and picosecond lasers," Analytical Chemistry, vol. 83, no. 1, pp. 6066, 2011.

[6] T. Streibel, J. Weh, S. Mitschke, and R. Zimmermann, "Thermal desorption/pyrolysis coupled with photoionization time-offlight mass spectrometry for the analysis of molecular organic 
compounds and oligomeric and polymeric fractions in urban particulate matter," Analytical Chemistry, vol. 78, no. 15, pp. 5354-5361, 2008.

[7] R. Zimmermann, F. Mühlberger, K. Fuhrer, M. Gonin, and W. Welthagen, "An ultracompact photo-ionization time-of-flight mass spectrometer with a novel vacuum ultraviolet light source for on-line detection of organic trace compounds and as a detector for gas chromatography," Journal of Material Cycles and Waste Management, vol. 10, no. 1, pp. 24-31, 2008.

[8] Q. Wang, Y. A. Dyakov, D. Wu et al., "Ionization/dissociation processes of methyl-substituted derivates of cyclopentanone in intense femtosecond laser field," Chemical Physics Letters, vol. 586, pp. 21-28, 2013.

[9] A. Li, T. Uchimura, H. Tsukatani, and T. Imasaka, "Trace analysis of polycyclic aromatic hydrocarbons using gas chromatography-mass spectrometry based on nanosecond multiphoton ionization," Analytical Sciences, vol. 26, no. 8, pp. 841-846, 2010.

[10] S. Yamaguchi, F. Kira, Y. Miyoshi et al., "Nearultraviolet femtosecond laser ionization of dioxins in gas chromatography/time-of-flight mass spectrometry," Analytica Chimica Acta, vol. 632, no. 2, pp. 229-233, 2009.

[11] O. Shitamichi, T. Matsui, Y. Hui, W. Chen, and T. Imasaka, "Determination of persistent organic pollutants by gas chromatography/laser multiphoton ionization/time-of-flight mass spectrometry," Frontiers of Environmental Science and Engineering in China, vol. 6, no. 1, pp. 26-31, 2012.

[12] A. Li, T. Imasaka, T. Uchimura, and T. Imasaka, "Analysis of pesticides by gas chromatography/multiphoton ionization/mass spectrometry using a femtosecond laser," Analytica Chimica Acta, vol. 701, no. 1, pp. 52-59, 2011.

[13] E. Sekreta, K. G. Owens, and J. P. Reilly, "Intensity-dependent laser ionization experiments involving the ${ }^{1} b_{1 u}$ state of benzene," Chemical Physics Letters, vol. 132, no. 4-5, pp. 450-455, 1986.

[14] N. Kirihara, H. Yoshida, M. Tanaka et al., "Development of a rimmpa-tofms: isomer selective soft ionization of PCDDs/DFs," Organohalogen Compound, vol. 66, pp. 731-738, 2004.

[15] T. Itoh, T. Uchimura, T. Uchida, M. Kawano, and T. Imasaka, "GC-MPI-MS of pentachlorodibenzofurans in flue gas using a UV picosecond laser," Chromatographia, vol. 68, no. 1-2, pp. 8994, 2008.

[16] M. van den Berg, L. S. Birnbaum, M. Denison et al., "The 2005 World Health Organization reevaluation of human and mammalian toxic equivalency factors for dioxins and dioxinlike compounds," Toxicological Sciences, vol. 93, no. 2, pp. 223241, 2006.

[17] O. P. Haefliger and R. Zenobi, "Laser mass spectrometric analysis of polycyclic aromatic hydrocarbons with wide wavelength range laser multiphoton ionization spectroscopy," Analytical Chemistry, vol. 70, no. 13, pp. 2660-2665, 1998.

[18] S. L. Murov, I. Carmichael, and G. L. Hug, Handbook of Photochemistry, Marcel Dekker, New York, NY, USA, 1993.

[19] I. B. Berlman, Handbook of Fluorescence Spectra of Aromatic Molecules, Academic Press, New York, NY, USA, 1971.

[20] A. Gutierrez-Llorente, R. Perez-Casero, B. Pajot et al., "Growth of anthracene thin films by matrix-assisted pulsed-laser evaporation," Applied Physics A: Materials Science and Processing, vol. 77, no. 6, pp. 785-788, 2003.

[21] A. Majcherczyk, C. Johannes, and A. Hüttermann, "Oxidation of polycyclic aromatic hydrocarbons (PAH) by laccase of
Trametes versicolor," Enzyme and Microbial Technology, vol. 22, no. 5, pp. 335-341, 1998.

[22] J. L. Newsted and J. P. Giesy, "Predictive models for photoinduced acute toxicity of polycyclic aromatic hydrocarbons to Daphnia magna, strauss (cladocera, crustacea)," Environmental Toxicology and Chemistry, vol. 6, no. 6, pp. 445-461, 1987.

[23] J. B. Birks, Photophysics of Aromatic Molecules, Wiley, New York, NY, USA, 1963.

[24] A. Samanta, C. Devadoss, and R. W. Fessenden, "Picosecond time-resolved absorption and emission studies of the singlet excited states of acenaphthylene," Journal of Physical Chemistry, vol. 94, no. 18, pp. 7106-7110, 1990.

[25] B. F. Plummer, M. J. Hopkinson, and J. H. Zoeller, "Dual wavelength fluorescence from acenaphthylene and derivatives in fluid media," Journal of the American Chemical Society, vol. 101, no. 22, pp. 6779-6781, 1979.

[26] Z. Wang, S. J. Weininger, and W. G. McGimpsey, "Photochemistry of the $\mathrm{T}_{2}$ state of anthracene," Journal of Physical Chemistry, vol. 97, no. 2, pp. 374-378, 1993.

[27] C. W. Wilkerson Jr., S. M. Colby, and J. P. Reilly, “Determination of polycyclic aromatic hydrocarbons using gas chromatography/laser ionization mass spectrometry with picosecond and nanosecond light pulses," Analytical Chemistry, vol. 61, no. 23, pp. 2669-2673, 1989.

[28] D. Helmig and W. P. Harger, "OH radical-initiated gas-phase reaction products of phenanthrene," Science of the Total Environment, vol. 148, no. 1, pp. 11-21, 1994. 

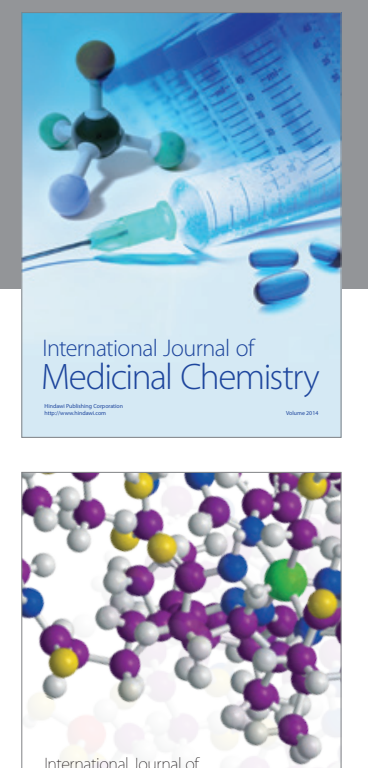

\section{Carbohydrate} Chemistry

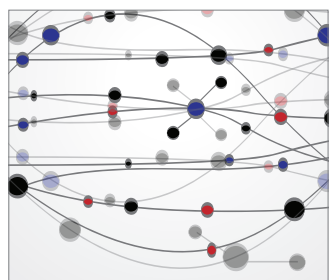

The Scientific World Journal
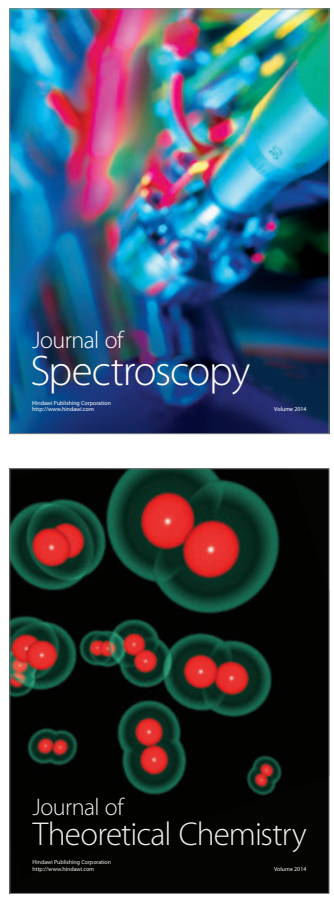
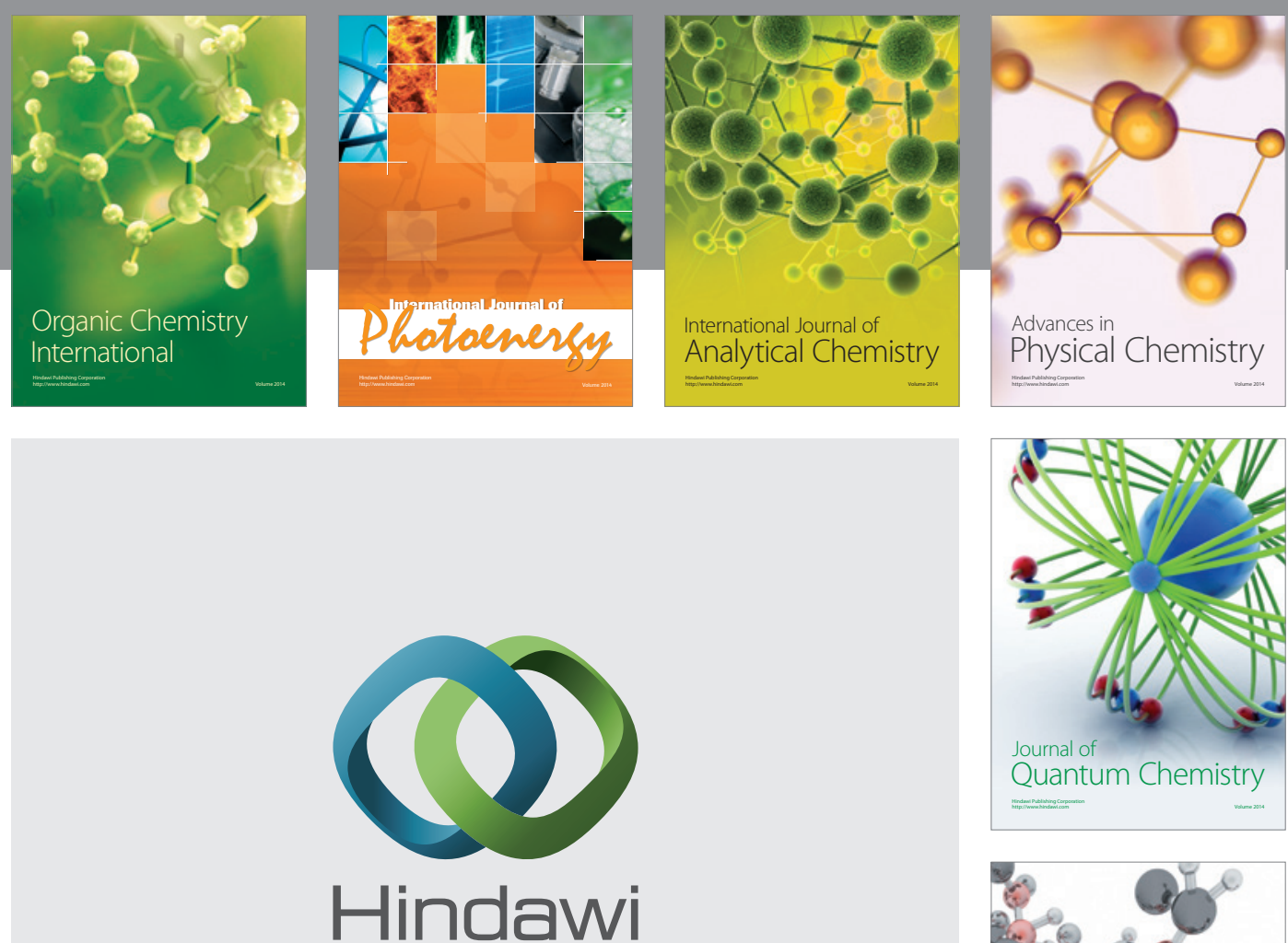

Submit your manuscripts at

http://www.hindawi.com

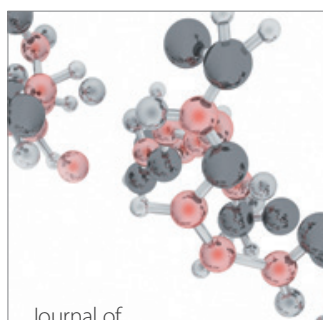

Analytical Methods

in Chemistry

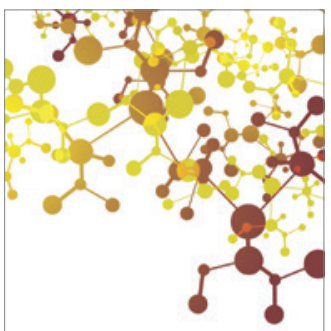

Journal of

Applied Chemistry



Inorganic Chemistry
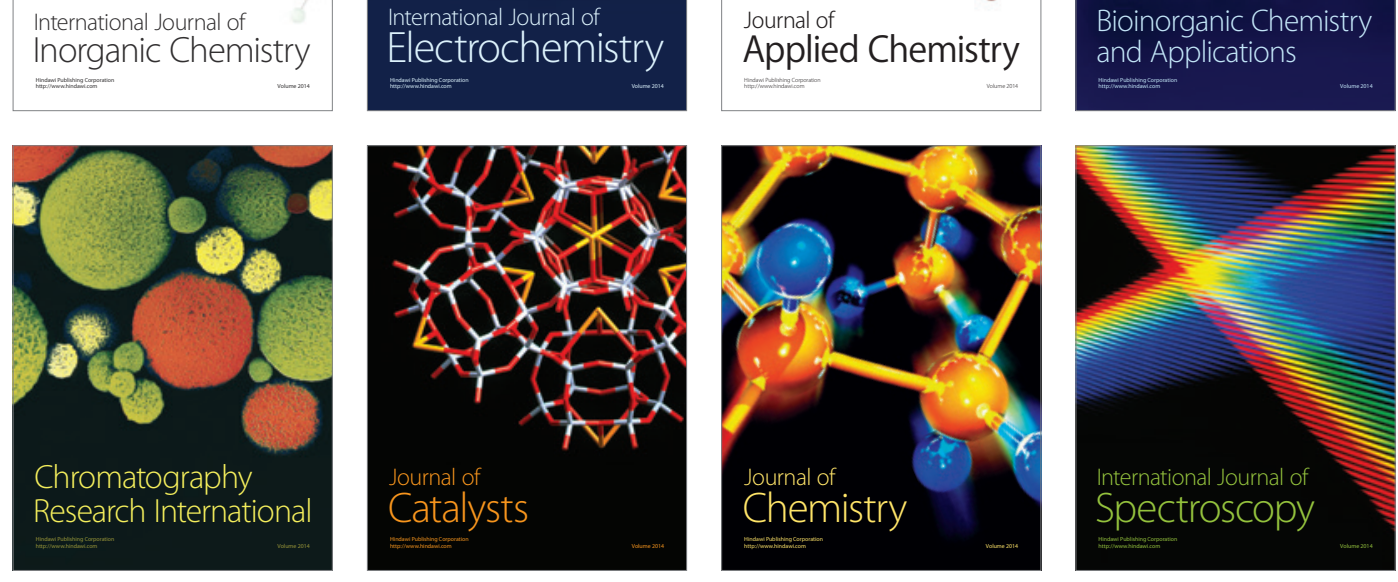